\title{
Identification of immunodominant IgE epitopes of the major house dust mite allergen Der f 24
}

\author{
ZE-LANG CAI* ${ }^{*}$ ZHEN ZHANG* ${ }^{*}$ WEN-LI LUO, YI-BO HOU, YONG-SHEN HE, JIA-JIE CHEN and KUNMEI JI \\ Department of Biochemistry and Molecular Biology, Health Science Center of \\ Shenzhen University, Shenzhen, Guangdong 518060, P.R. China
}

Received June 5, 2019; Accepted August 16, 2019

DOI: $10.3892 /$ ijmm.2019.4345

\begin{abstract}
Previously, a ubiquinol-cytochrome c reductase binding protein (UQCRB) homolog was identified in the house dust mite (HDM) species Dermatophagoides farinae (Der f) as a major allergen. In the present study, the immunodominant immunoglobulin E ( $\mathrm{IgE}$ ) epitope of the protein Der f 24 was investigated. Analysis of the homologous amino acid (aa) sequences in Der $f$ and human UQCRB was performed. Four different recombinant Der f 24 and hybrid proteins formed by integrating Der f and human UQCRB sequences were expressed in Escherichia coli, purified using Ni-NTA resins, and IgE-binding activity was determined using IgE-western blotting and enzyme-linked immunosorbent assay (ELISA) experiments. IgE epitopes were further identified by IgE-dot blotting and IgE-ELISA with synthetic polypeptides and HDM-allergic sera. Three-dimensional (3D) structural modeling was used to analyze the position of the immunodominant IgE epitope. The amino acid sequence homology between Der f 24 and the human UQCRB protein was determined to be $39.34 \%$. IgE-ELISA and western blot analysis showed that all of the Der f-human UQCRB hybrid proteins generated, except for the one lacking 59 residues of the N-terminal region of Der f 24, were bound by allergic serum IgE. A synthetic polypeptide consisting of 32 residues of the $\mathrm{N}$-terminal reacted with IgEs from HDM-allergic sera and
\end{abstract}

Correspondence to: Dr Jia-Jie Chen or Professor Kunmei Ji, Department of Biochemistry and Molecular Biology, Health Science Center of Shenzhen University, 1066 Xilixuanyuan Road, Nanshan, Shenzhen, Guangdong 518060, P.R. China

E-mail: chenjj@szu.edu.cn

E-mail: jkm@szu.edu.cn

${ }^{*}$ Contributed equally

Abbreviations: HDM, house dust mite; Der f 24, group 24 allergen of Dermatophagoides farina; UQCRB, ubiquinol-cytochrome c reductase binding protein; IgE, immunoglobulin E; ELISA, enzyme-linked immunosorbent assay; IEDB, immune epitope database

Key words: house dust mite; Der f 24; B cell epitope; IgE-binding could be used to generate high titer specific IgG or specific IgE antibodies in immunized mice. The 32-aa N-terminal region of Der $\mathrm{f} 24$ was localized to a structural protrusion, which may facilitate specific IgE-binding. These results indicate that the immunodominant IgE epitope of Der f 24 is located mainly in a 32-residue region of the N-terminus. These findings may inform the mechanisms of HDM allergy sensitization and allergy immunotherapy development.

\section{Introduction}

In recent years, there has been an increase in the prevalence of and financial burden associated with allergic disorders, including bronchial asthma, allergic rhinitis and atopic dermatitis (1). Remarkably, more than half of diagnosed allergic diseases are attributed to house dust mite (HDM) allergens (2). The HDM species Dermatophagoides pteronyssinus (Der p) and Dermatophagoides farinae (Der f) are the main indoor sources of inhaled allergens triggering IgE-mediated anaphylactic reactions. HDM is the most common cause of respiratory allergy worldwide. (2). The rate of HDM allergy is high in developed countries; previous clinical studies showed that HDM allergic sensitization is $>20 \%$ in Europe (3) and $\leq 40 \%$ in North America (4). In-depth analysis of HDM allergens is required to elucidate the mechanism of HDM allergen sensitization and to inform the development of diagnosis and treatment for HDM allergies (5).

Of the 39 HDM allergen groups that have been identified (6), Group I and Group II HDM allergen reactivity are the most prevalent, followed by Group 23 and Group 24 (7-9). Hence, Group I and II HDM allergens are considered to be the major allergens of HDM. Cysteine protease activity of the Group I HDM allergen Der p 1 has been shown to enhance the immunoglobulin E (IgE) antibody response selectively (10), and Der $\mathrm{p} 1$ proteolytic activity leads to augmented IgE reactivity to Der $\mathrm{p} 1$ itself as well as to other allergens in the microenvironment (11). Furthermore, Der p 2 has been shown to induce inflammatory allergenic effects via binding of Toll-like receptor 4 (TLR4) $(12,13)$. Thus, Group 1 and 2 HDM allergens exhibit IgE-binding activity with sera from most HDM-allergic patients (14) and have been shown to induce $\mathrm{T}$ helper type 2 (Th2) immune responses through cysteine protease functions (15) and facilitation of TLR4 signaling (12), respectively. However, the mechanisms mediating the allergic 
reactions triggered by most HDM allergens, which may vary substantially from group to group, have not yet been resolved.

$\mathrm{IgE}$ interaction with exogenous allergens promotes mast cell degranulation, which induces inflammation (16). The domain of each allergen bound by serum IgEs are known as B cell IgE-binding epitopes (17). Analysis of B cell IgE epitopes can provide good indicators for allergy diagnosis, prediction of the clinical severity of allergic diseases and monitoring of the development of allergen tolerance (18). B cell epitopes for Group 1, 2, 3, 7, 11, 13 and 33 HDM allergens have been identified with peptide-displaying phage and artificial synthetic peptide scanning technologies (19-23). In our previously study, the Group 24 HDM allergen, Der f 24, was found to be a major HDM allergen and to function as a ubiquinol cytochrome $\mathrm{c}$ reductase binding protein (UQCRB) homolog (9). In a multitude of species, UQCRB proteins play an important role in the maintenance of mitochondrial complex III for electron transport and cellular oxygen sensing (24). The only UQCRB proteins that have been reported to exhibit allergenic activity are those from Der $f$ and Der $p$ (25). The Der $f$ and Der $p$ mite species contribute differently to HDM induced allergic disease, potentially due to their differing geographical distributions (26) or inherent characteristic differences between them $(18,27)$. Importantly, it has been shown previously that Der p 24 exhibits strong IgE-binding activity via an immunodominant IgE epitope in its N-terminal 32-residue region (25); however, the dominant IgE epitope of Der f 24 may not be the same as that of its homolog Der p 24, particularly given the differing protein sequences of the two allergens. It remains to be determined how the allergenic properties of the UQCRB protein in Der $f$ differs from and/or resembles the Der $p$ UQCRB protein.

Detailed knowledge about the epitopes responsible for IgE-binding of allergenic peptides can help to inform allergy diagnosis and prognosis, and may facilitate the rational design of hypoallergenic candidate immunotherapeutic vaccines. The Der f 24 UQCRB protein homolog shows strong IgE reactivity with serum from HDM-allergic patients in vitro and in vivo, indicating that Der f 24 may play a key role in HDM sensitization, and thus, may have potential for the improvement of diagnosis and treatment (9).

The mechanism by which UQCRB protein homologs affect allergic reactions has not been resolved, and an analysis of the immunodominant IgE epitope of Der f 24 has yet to be reported. Therefore, the aim of the present study was to identify and characterize the IgE-binding epitope of Der f 24 using IgE-western blotting, enzyme-linked immunosorbent assays (ELISAs) and IgE-dot blotting. The IgE epitope immunodominance of Der f 24 was explored with IgE-binding activity assays with Der f-human UQCRB hybrid proteins. Finally, homology modeling was used to predict the structural position of the IgE-binding site within Der f 24.

\section{Materials and methods}

Serum samples. Serum samples from 30 individuals with HDM allergy (13 males and 17 females; age range, $18-55$ years) and 24 non-allergic individuals (10 males and 14 females; age range, 18-55 years) were obtained from the First Affiliated Hospital of Guangzhou Medical College (Guangdong, China). The
Table I. Clinicopathological characteristics of patients with HDM allergy.

\begin{tabular}{clccc}
\hline $\begin{array}{l}\text { Patient } \\
\text { ID }\end{array}$ & Sex & $\begin{array}{c}\text { Age, } \\
\text { years }\end{array}$ & $\begin{array}{c}\text { ImmunoCAP } \\
\text { HDM-specific IgE test }\end{array}$ & $\begin{array}{c}\text { Clinical } \\
\text { history }\end{array}$ \\
\hline 1 & Female & 23 & 3 & - \\
2 & Female & 18 & 3 & BA \\
3 & Male & 35 & 3 & AR \\
4 & Female & 51 & 4 & BA \\
5 & Male & 29 & 4 & AR \\
6 & Female & 36 & 4 & AR \\
7 & Male & 52 & 4 & AR \\
8 & Male & 19 & 5 & BA \\
9 & Female & 47 & 5 & AR+BA \\
10 & Male & 28 & 5 & - \\
11 & Male & 34 & 5 & AR+BA \\
12 & Female & 53 & 6 & AR \\
13 & Female & 21 & 6 & BR+BA \\
14 & Male & 43 & 6 & AR+BA \\
15 & Female & 55 & 6 &
\end{tabular}

agE levels were defined as follows: Level 3, 3.5-17.5 IU/ml; level 4, 17.5-50.0 IU/ml; level 5, 50.0-100 IU/ml; and level 6, >100 IU $/ \mathrm{ml}$. HDM, house dust mite; IgE, immunoglobulin E; BA, bronchial asthma; AR, allergic rhinitis; -, data not shown.

allergic patient sample consisted entirely of individuals who had experienced anaphylaxis caused by HDM exposure and who had IgE levels $>3$, measured using a clinical ImmunoCAP allergen detection system (Phadia; Thermo Fisher Scientific, Inc.). Patient clinicopathological information, including sex, age, ImmunoCAP HDM-specific IgE levels and previous diagnoses are presented in Table I. Ethics approval for the current study was obtained from the First Affiliated Hospital of Guangzhou Medical College (approval no. 2012-51). Informed consent was obtained from all individual participants included in the study. All procedures involving human participants were in accordance with the ethical standards of the committee. Sera pooled from 10 patients with HDM allergy were used to detect the ability of IgE-binding to recombinant Der (rDer) $f$ 24 and hybrid proteins.

Animals. Female BALB/c mice (4-5 weeks old) were purchased from Guangdong Medical Laboratory Animal Center (Foshan, China), and housed in a specific pathogen-free environment with a relatively stable temperature $\left(24 \pm 1^{\circ} \mathrm{C}\right)$ and humidity $(55 \pm 10 \%)$ for $>1$ week before experimentation. All studies involving mice were approved by the Animal Ethical and Welfare Committee of the School of Medicine of Shenzhen University (Guangdong, China).

Analysis of sequence homology. The amino acid (aa) sequences of Der f 24 (GenBank no. AJK91617.1), Der p 24 (GenBank no. KP893174.1), human UQCRB (GenBank no. EAW91749) and mouse UQCRB (GenBank no.NM_026219) were obtained from the National Center for Biotechnology Information 
Table II. Summary of aa sequence constitution of hybrid proteins.

\begin{tabular}{|c|c|c|}
\hline Name & Aa sequence & No. aa \\
\hline Der f 24-Hyb1 & $\begin{array}{l}\text { MVHLTKTLRFINNPGFRKFYYGLQGYNKYGLYYDDFYDYT } \\
\text { DAAHLEAV RRLPPDLYDQHMFRIKRALDLNLKHQILPKE } \\
\text { QWTKYEEENFYLEPYLKEVIRERKEREEWAKK }\end{array}$ & 111 aa (mite,1-59 aa; human, 60-111 aa) \\
\hline Der f 24-Hyb2 & $\begin{array}{l}\text { MAGKQAVSASGKWLDGIRKWYYNAAGFNKLGLMRDDTI } \\
\text { YEDEDVKEAIRRLPENLYNDRTYRL VRASQLEITKQFLPKE } \\
\text { QWPSYEEDMDKGRFLTPYLDEVMKEKKEKEEWINFLSKD }\end{array}$ & 118 aa (human, $1-59$ aa; mite, 60-118 aa) \\
\hline Der f 24-Hyb3 & $\begin{array}{l}\text { MVHLTKTLRFINNPGFRKFYYGLQGYNKYGLYYDDFYD } \\
\text { YTDAAHLEAVRRLPPDLYDQHTYR LVRASQLEITKQFLPK } \\
\text { EQWTKYEEENFYLEPYLKEVIRERKEREEWAKK }\end{array}$ & 111 aa (mite, 1-78 aa; human, 79-111 aa) \\
\hline Der f 24-Hyb4 & $\begin{array}{l}\text { MVHLTKTLRFINNPGFRKFYYGLQGYNKYGLYYDDFYDYE } \\
\text { DEDVKEAIRRLPENLYNDRMFRIKRALDLNLKHQILPKEQ } \\
\text { WTKYEEENFYLEPYLKEVIRERKEREEWAKK }\end{array}$ & 111 aa (mite, 1-39 aa; human, 40-111 aa) \\
\hline
\end{tabular}

Der f 24, group 24 allergen of Dermatophagoides farina; aa, amino acid; Hyb, hybrid.

library (NCBI; https://www.ncbi.nlm.nih.gov/protein/) and imported into DNAMAN 8 software (version 8; Lynnon LLC) for homology comparison. FASTA-format sequences were obtained for further analysis.

Construction of vectors and expression and purification of hybrid proteins. Human UQCRB has not been reported to have $\operatorname{IgE}$ epitopes that induce an autoimmune response. Hybrid Der $f$ and human UQCRB protein sequences (Table II) were designed and the resultant cDNA sequences were synthesized by GenScript (Nanjing) Co. Ltd. The following synthetic genes were registered in the GenBank database: Der f 24-Hyb1 [mite, 1-59 aa; human, 60-111 aa (accession no. KP939229)]; Der f 24-Hyb2 [human, 1-59 aa; mite, 60-118 aa (accession no. KP939230)]; Der f 24-Hyb3 [mite, 1-78 aa; human, 79-111 aa (accession no. KP939231)]; and Der f 24-Hyb4 [mite, 1-39 aa; human, 40-111 aa (accession no. KP939232)]. These cDNAs were cloned into pET-His vectors (Miaoling Bioscience and Technology Co., Ltd.). The recombinant plasmids were confirmed by DNA sequencing, and for protein expression, $100 \mathrm{ng}$ DNA was transformed into Escherichia coli BL21 (DE3) pLysS competent cells (Novagen; Merck KGaA) by heat shock. The expression and purification of recombinant protein was performed as described previously (28). The rDer f 24 protein and the hybrid proteins were isolated in the form of inclusions. They were purified by Ni-NTA gel affinity chromatography (GE Healthcare) and subjected to IgE-western blotting or IgE-dot blotting. rDer f 24 protein quality was evaluated by determining the activity of IgE-binding with HDM-allergic sera, and required a positive rate of $\sim 50 \%$ using 10 individual HDM-allergic sera in IgE-ELISA (9). The recombinant protein concentrations were determined using the Bradford method (Bio-Rad Laboratories, Inc.). The recombinant proteins (40 $\mu \mathrm{g} / \mathrm{well})$ were analyzed using sodium dodecyl sulfate-polyacrylamide gel electrophoresis (SDS-PAGE) and the gel was stained with Coomassie Brilliant Blue R-250 Staining Solution (Bio-Rad Laboratories, Inc.).
Synthesis of overlapping polypeptides. Five overlapping Der $\mathrm{f} 24$ polypeptides (31-33 aa) were designed and synthesized by Shanghai Qiang Yao Biotechnology Co. Ltd. The overlapping regions were 10 aa long. The polypeptides were coupled with bovine serum albumin (BSA; Amresco, LLC) or ovalbumin (OVA; Sigma-Aldrich; Merck KGaA) at the cysteine residue of the $\mathrm{N}$ - or $\mathrm{C}$-terminus using a 3-maleimidobenzoic acid N-hydroxysuccinimide method, which was performed by Shanghai Qiang Yao Biotechnology Co. Ltd.

IgE western blotting and IgE dot blotting. rDer f 24 and hybrid proteins were separated by $12 \%$ SDS-PAGE and transferred to polyvinylidene difluoride (PVDF) membranes (EMD Millipore) for western blotting. For dot blotting, $2 \mu \mathrm{l}$ rDer f 24 (concentration $1 \mu \mathrm{g} / \mu \mathrm{l}$ ) and synthetic polypeptides (BSA-1-32 aa, BSA-23-54 aa, BSA-45-76 aa, BSA-67-98 a and BSA-89-118 aa) were dotted onto PVDF membranes. The membranes were blocked with 5\% Difco ${ }^{\mathrm{TM}}$ skim milk (DSM; BD Biosciences) diluted in Tris buffered saline containing $0.05 \%$ Tween-20 at $4^{\circ} \mathrm{C}$ overnight. Western blot membranes were incubated with a mixture of allergic sera from different 10 patients with HDM allergy (1:10 dilution) for $2 \mathrm{~h}$ at $37^{\circ} \mathrm{C}$. For dot blotting, a mix of 10 serum samples from HDM-allergic patients with high IgE-binding to rDer f 24 and a mix of 3 non-allergic sera samples (1:10 dilution) were incubated for $2 \mathrm{~h}$ at $37^{\circ} \mathrm{C}$. Following washing, the membranes were incubated with horse radish peroxidase-conjugated mouse anti-human $\operatorname{IgE}$ antibody (1:2,000 dilution; cat. no. 9160-05; SouthernBiotech) for $1.5 \mathrm{~h}$ at $37^{\circ} \mathrm{C}$. Antigen-antibody complexes on membranes were visualized using a Pierce ${ }^{\mathrm{TM}} 3^{\prime}$-diaminobenzidine substrate kit (Thermo Fisher Scientific, Inc.).

$I g E$-ELISA. rDer f 24 protein and conjugated polypeptides (200 ng/well; diluted in carbonate buffer; $\mathrm{pH} 9.6$ ) were coated onto 96-well plates, and incubated at $4^{\circ} \mathrm{C}$ overnight. The plates were blocked with $5 \%$ DSM in phosphate-buffered saline containing $0.05 \%$ Tween-20 (PBST) for $3 \mathrm{~h}$ at $37^{\circ} \mathrm{C}$. Washed plates were incubated with sera (1:10 dilution) from 15 


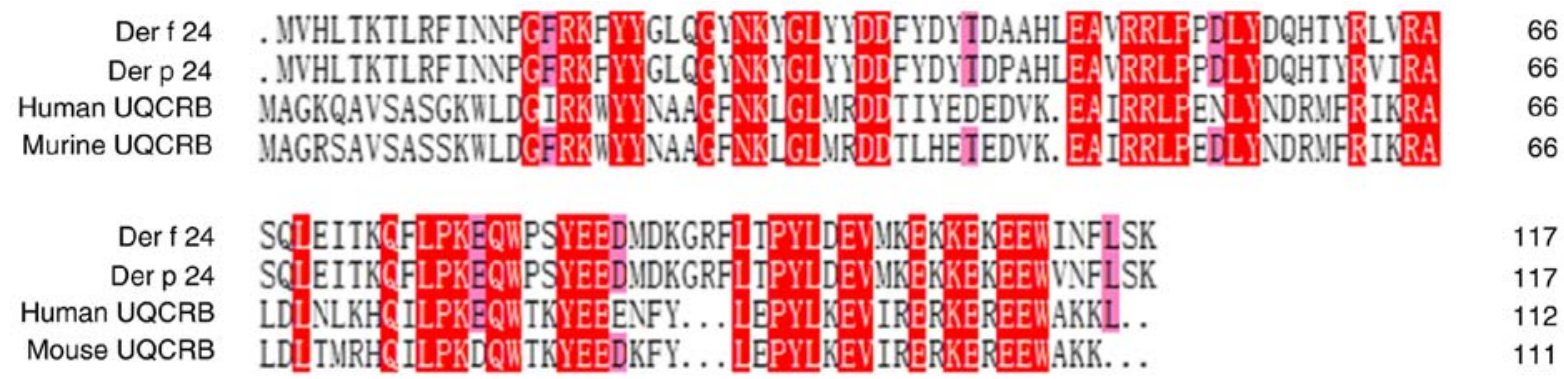

Figure 1. Homology between Der f 24 and human UQCRB. Identical residues are shaded in red. The amino acid sequences of Der f 24 (GenBank no. AJK91617.1), Der p 24 (GenBank no. KP893174.1), human UQCRB (GenBank no. EAW91749) and mouse UQCRB (GenBank no. NM 026219) were imported into DNAMAN 8 software for homology comparison. Der f 24, group 24 allergen of Dermatophagoides farina; Der p 24, group 24 allergen of Dermatophagoides pteronyssinus; UQCRB, ubiquinol-cytochrome c reductase binding 1 protein.

A

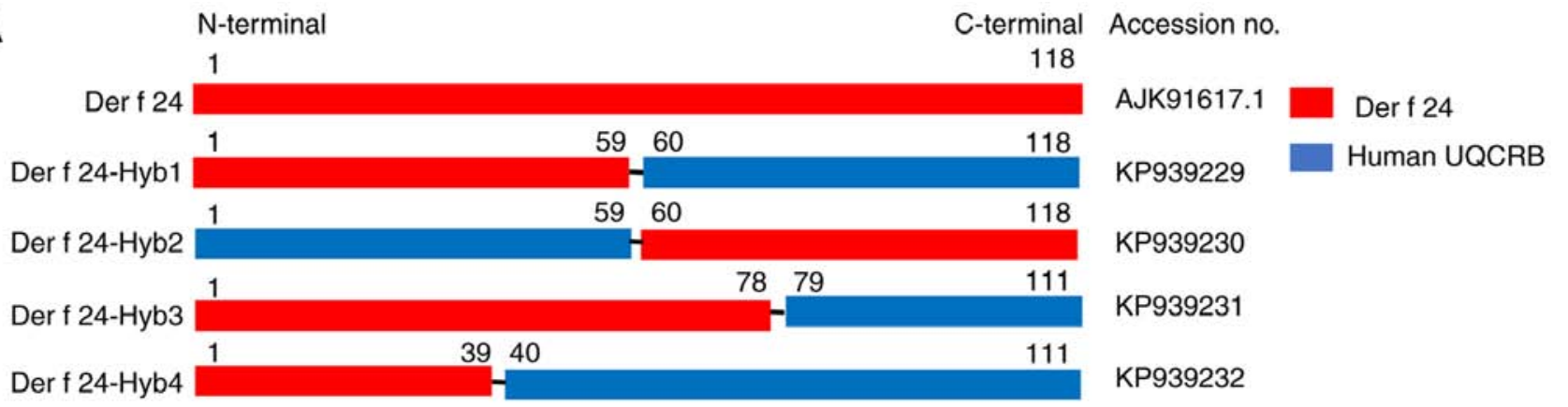

B
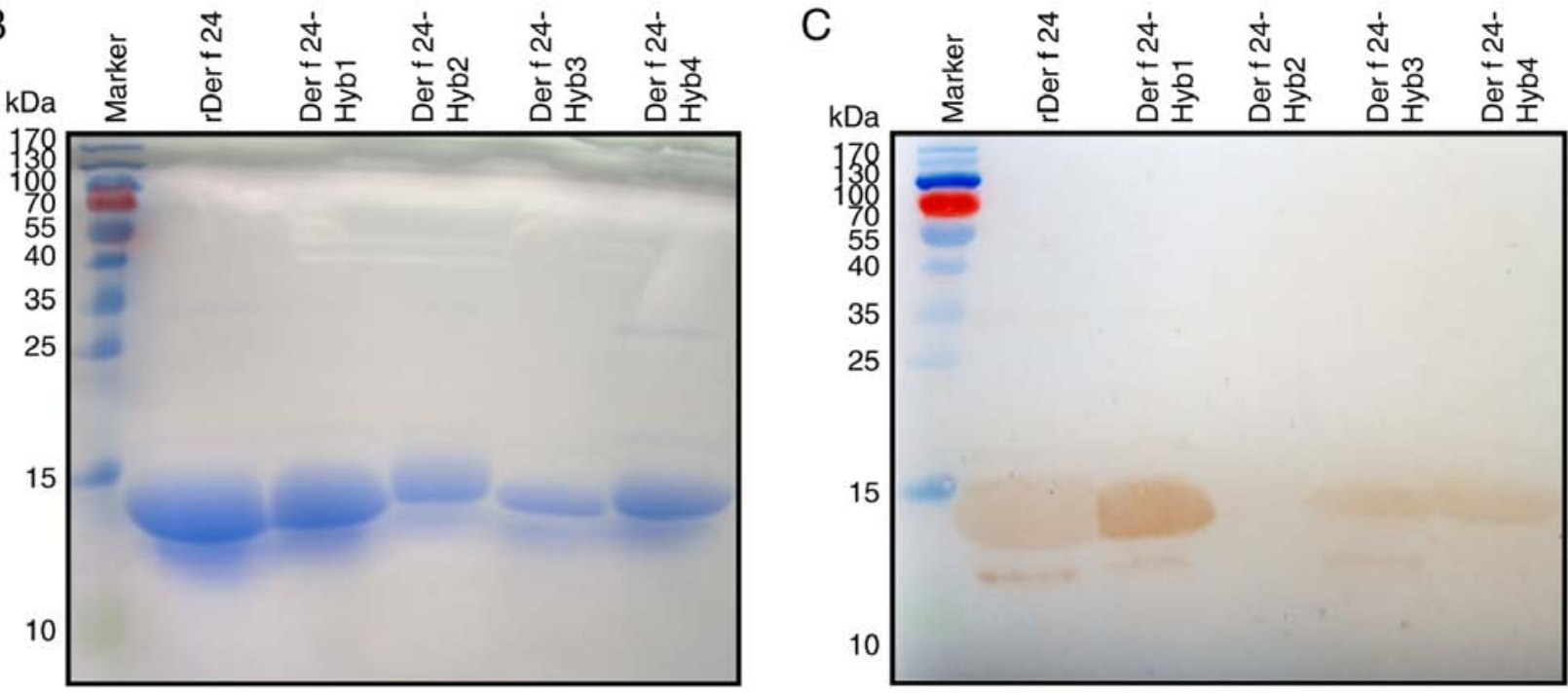

Figure 2. Construction, purification and IgE-binding activity of rDer f 24 and hybrid proteins. (A) Schematic diagram of rDer f 24 and hybrid proteins. (B) SDS-PAGE analysis of purified rDer f 24 and hybrid proteins with Coomassie Brilliant Blue staining. (C) Western blot analysis using sera pooled from 10 patients with HDM allergy showing IgE-binding of rDer f 24 and its hybrid proteins. Der f 24, group 24 allergen of Dermatophagoides farina; rDer f 24, recombinant Der f 24; IgE, immunoglobulin E; UQCRB, ubiquinol-cytochrome c reductase binding 1 protein; Hyb, hybrid; HDM house dust mite.

HDM-allergic patients and 15 non-allergic individuals for $2 \mathrm{~h}$ at $37^{\circ} \mathrm{C}$. Serum IgEs were then detected by incubating with a mouse anti-human IgE horseradish peroxidase-conjugated antibody $\left(1: 2,000\right.$ dilution; SouthernBiotech) for $1.5 \mathrm{~h}$ at $37^{\circ} \mathrm{C}$. The plates were washed with PBST buffer. The reaction was detected by adding $100 \mu 1$ 3,3,5,5'-tetramethylbenzidine substrate $(1 \mathrm{mM}$; Invitrogen; Thermo Fisher Scientific, Inc.), and absorbance was measured at $450 \mathrm{~nm}$ using a microplate reader (Bio-Rad Laboratories, Inc.). After screening, the 15 HDM allergic sera all displayed positive reaction to allergen Der $\mathrm{f} 24$.
Sensitization of mice. Female BALB/c mice (6-8 weeks of age; $17-20 \mathrm{~g}$ in weight) were purchased from Guangzhou Experimental Animal Center (Guangdong, China). The mice $(\mathrm{n}=3)$ were sensitized by intraperitoneal injection of $50 \mu \mathrm{g}$ OVA or OVA-1-32 aa conjugate with Imject ${ }^{\mathrm{TM}}$ Alum adjuvant (2:1 $\mathrm{v} / \mathrm{v}$ in total volume $200 \mu \mathrm{l}$; Thermo Fisher Scientific, Inc.). On day 21 after injection, the mice were euthanized by cervical dislocation under deep isoflurane anesthesia and the sera were collected. Serum levels of specific IgE and IgG targeting the BSA-1-32 conjugate were measured by ELISA as described 
A N-terminal

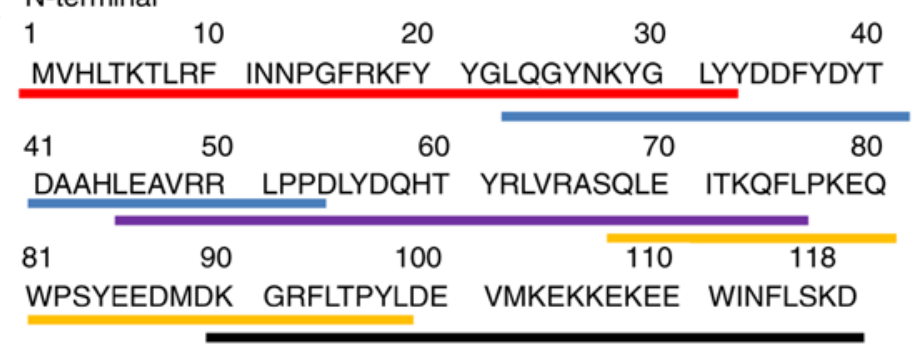

C-terminal

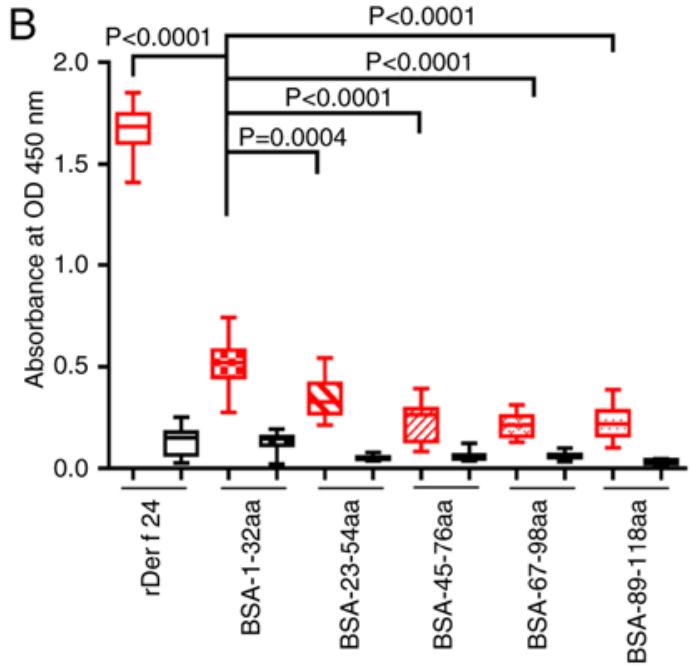

D

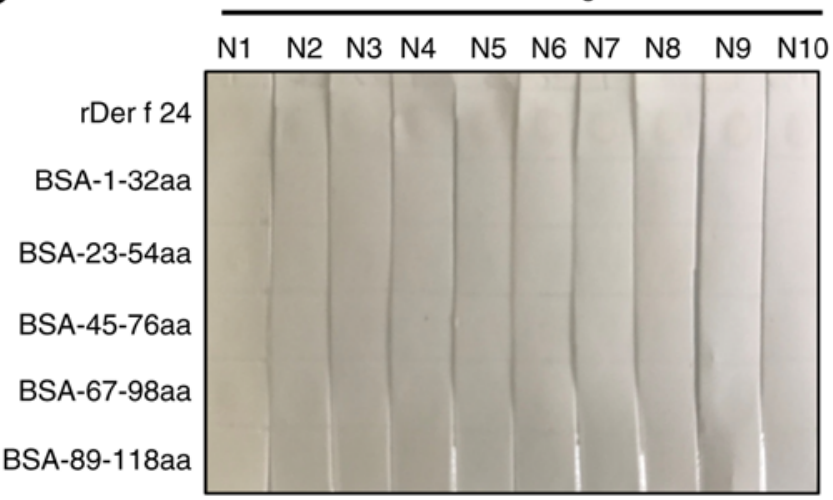

C
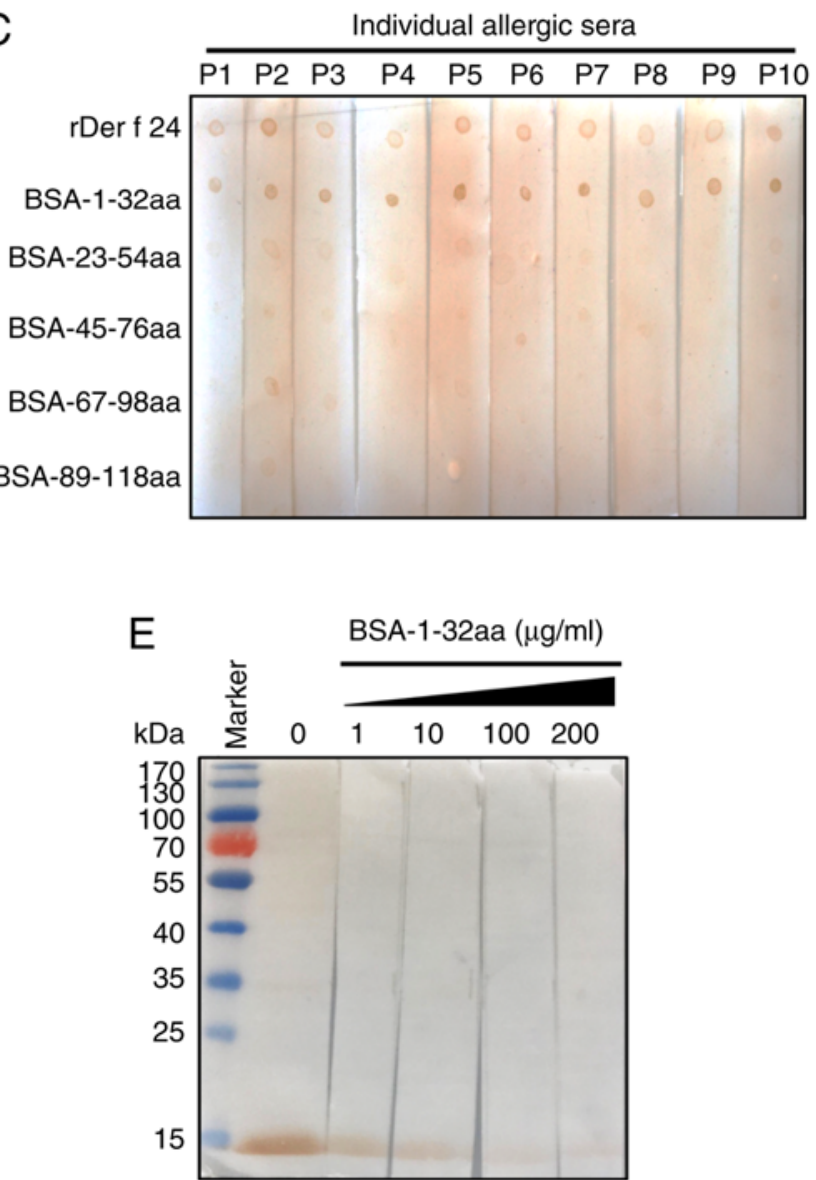

Figure 3. Comparative analysis of IgE-binding reactivity of rDer $\mathrm{f} 24$ and synthetic peptides. (A) Schematic diagram showing analogous locations of synthetic peptides in Der f 24. These peptides were coupled with BSA at the N- or C-terminus. (B) Indirect ELISA of rDer f 24 and synthetic peptides with 15 individual sera (red) from HDM allergic patients and 15 non-allergic controls (black) to determine IgE-binding activity. $\mathrm{P}<0.05$ was considered significant. (C and D) Dot blots of rDer $\mathrm{f} 24$ and synthetic peptides with pooled sera above from (C) $10 \mathrm{HDM}$-allergic individuals or (D) 10 individual non-allergic controls. (E) Competitive IgE western blotting of Der $\mathrm{f} 24$ with or without BSA-1-32 aa antigen confirming the IgE-binding capacity of the Der $\mathrm{f} 24$ 1-32 aa peptide. The sera pool of 15 HDM allergic individuals was used and the BSA-1-32 aa conjugate was the inhibitory antigen at different concentrations. Der $\mathrm{f} 24$, group 24 allergen of Dermatophagoides farina; $r$ Der f 24, recombinant Der f 24; IgE, immunoglobulin E; HDM, house dust mite; aa, amino acid.

previously (29). The health status of the experimental mice was monitored twice daily and humane endpoints were used to determine if the mice met criteria to be euthanized. These criteria included weight loss $>10-15 \%$, lethargy, inability to stand and anorexia. Mice that met the designated criteria were euthanized by cervical dislocation under deep isoflurane anesthesia.

RBL-2H3 degranulation assay. The rat mast cell leukemia cell line, RBL-2H3 (Guangzhou Cellcook Biotech Co., Ltd.) was cultured in complete Dulbecco's modified eagle medium with 4.0 mM L-glutamine, sodium pyruvate penicillin (100 U/ml),
$100 \mu \mathrm{g} / \mathrm{ml}$ streptomycin, non-essential amino acids and $10 \%$ fetal bovine serum (Gibco; Thermo Fisher Scientific, Inc.) in a humidified incubator at $37^{\circ} \mathrm{C}$ and $5 \% \mathrm{CO}_{2}$. A cell degranulation assay was performed as previously described (30). The RBL-2H3 mast cell cells were seeded in 96-well plates for $18 \mathrm{~h}$. After washing three times using Tyrode's buffer (Beijing Solarbio Science \& Technology, Co., Ltd.), cells were exposed to sera of sensitized mice for $2 \mathrm{~h}$ and then stimulated with $10 \mu \mathrm{g} / \mathrm{ml}$ BSA-1-32aa conjugate for $45 \mathrm{~min}$. Degranulation was assessed by measurement of $\beta$-hexosaminidase release into the supernatant and of unreleased enzyme in the respective cell lysate. 

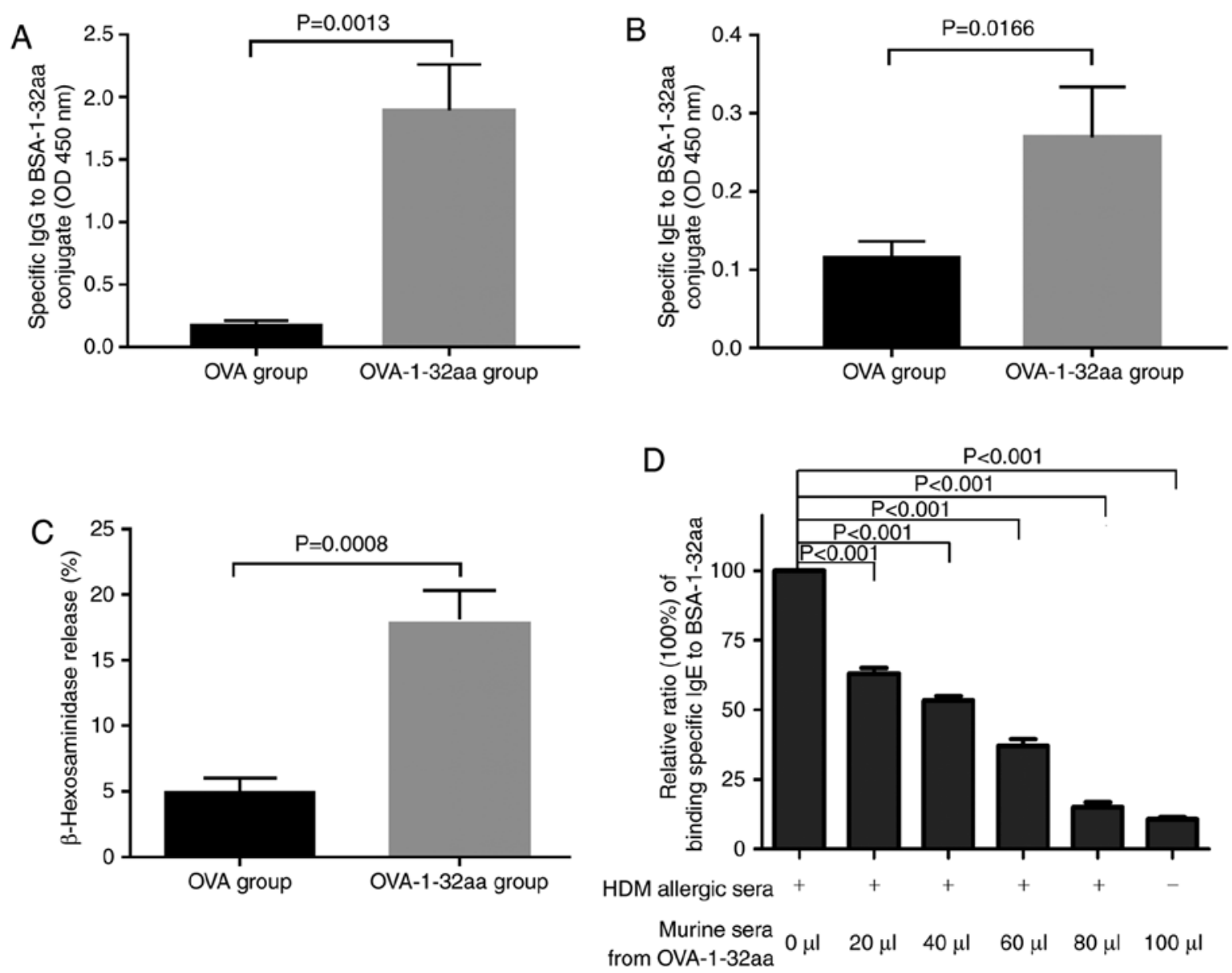

Figure 4. Peptide 1-32 aa domain of Der $\mathrm{f} 24$ produces high antibody titers of specific $\operatorname{IgE}$ in sensitized mice. Concentrations of (A) IgG and (B) IgE against Der f 24 1-32 aa peptide in sera from mice sensitized with OVA- and OVA-1-32 aa conjugate. (C) Release of $\beta$-hexosaminidase from the mast cell line RBL-2H3 treated with sera from mice sensitized with OVA- and OVA-1-32 aa conjugate, and subsequently exposed to $10 \mu \mathrm{g} / \mathrm{ml}$ BSA-1-32 aa conjugate. The experiments were performed with serum samples from sensitized mice $(\mathrm{n}=3)$. (D) Competitive IgE-ELISA to test binding of the BSA-1-32 aa conjugate with sera derived from mice sensitized with OVA-1-32 aa and sera derived from individuals with HDM allergy. Der f 24, group 24 allergen of Dermatophagoides farina; IgG, immunoglobulin G; IgE immunoglobulin E; aa, amino acid; HDM, house dust mite; OVA, ovalbumin; HDM, house dust mite.

Der $f 243 D$ structural modeling. The structure of cytochrome b-c1 complex subunit 7 (QCR7, also known as UQCRB) template from Bos Taurus was obtained from the RCSB Protein Data Bank (http://www.rcsb.org/; PDB code, 5klv.1), and was used as template for 3D modeling of the Der $\mathrm{f} 24$ protein. The 3D structure of Der f 24 was modeled in SWISS-MODEL online software (http://swissmodel.expasy.org/) based on the bovine QCR7 template. Ramachandran plots and root mean square deviation (RMSD) structure assessment methods were used to assess the reliability of the predicted 3D model using the PDBsum database (http://www.ebi.ac.uk/pdbsum/) which specializes in structural analyses and pictorial representations of models. The loci of 5 synthetic polypeptides in the Der $\mathrm{f} 24$ protein were indicated with color-coding for IgE-binding activity analysis. The key residues in the 1-32 aa domain region of Der f 24 were evaluated using the Immune epitope database (IEDB) prediction online tool (https://www.iedb.org/) (31). Amino acid property scales for predicting antigenic determinants were developed using the BepiPred Linear Epitope Prediction tool (version 1.0; http://www.cbs.dtu.dk/services/bepipred/).

Statistical analysis. Experimental data are presented as mean \pm SD. Statistical significance was calculated using
GraphPad Prism 7 (GraphPad Software, Inc.). Group differences were determined by analysis of variance followed by Dunnett's test for multiple comparisons. $\mathrm{P}<0.05$ were considered to indicate a statistically significant difference.

\section{Results}

Low sequence homology of UQCRB protein between Der $f$ and Homo sapiens. The Der f 24 gene encodes a protein sequence of 118 aa and is registered in the NCBI library. As presented in Fig. 1, a homology comparison in DNAMAN 8 showed Der f 24 was highly homologous to Der p 24 (96.61\%). Human UQCRB protein was highly homologous to mouse UQCRB (86.61\%). However, low aa homology was observed between Der f 24 and the human UQCRB protein (39.34\%).

IgE-binding of Der $f 24$ and hybrid proteins. Pooled samples of serum from patients with HDM allergy were found to have improved IgE specificity compared to individual samples in preliminary experiments. Therefore, pooled serum samples were used for screening the immunodominant IgE epitope of the allergen. The Der $f$ and human components of purified $r$ Der f 24, hybrid Der f proteins and human UQCRB protein are 

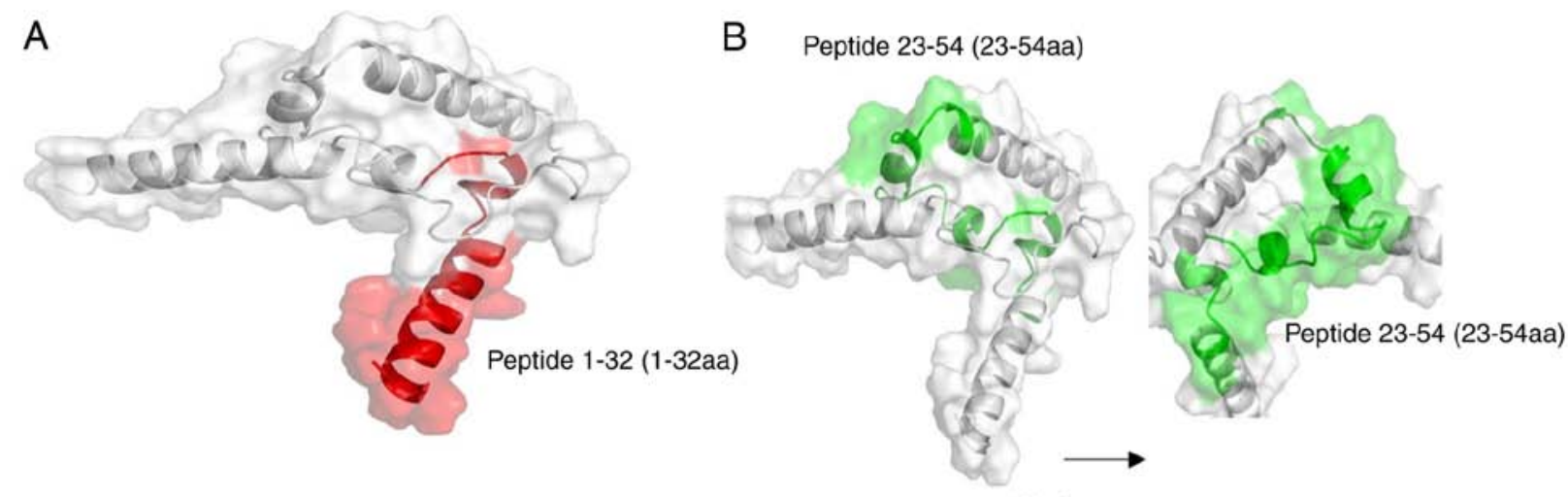

180 degree turnover
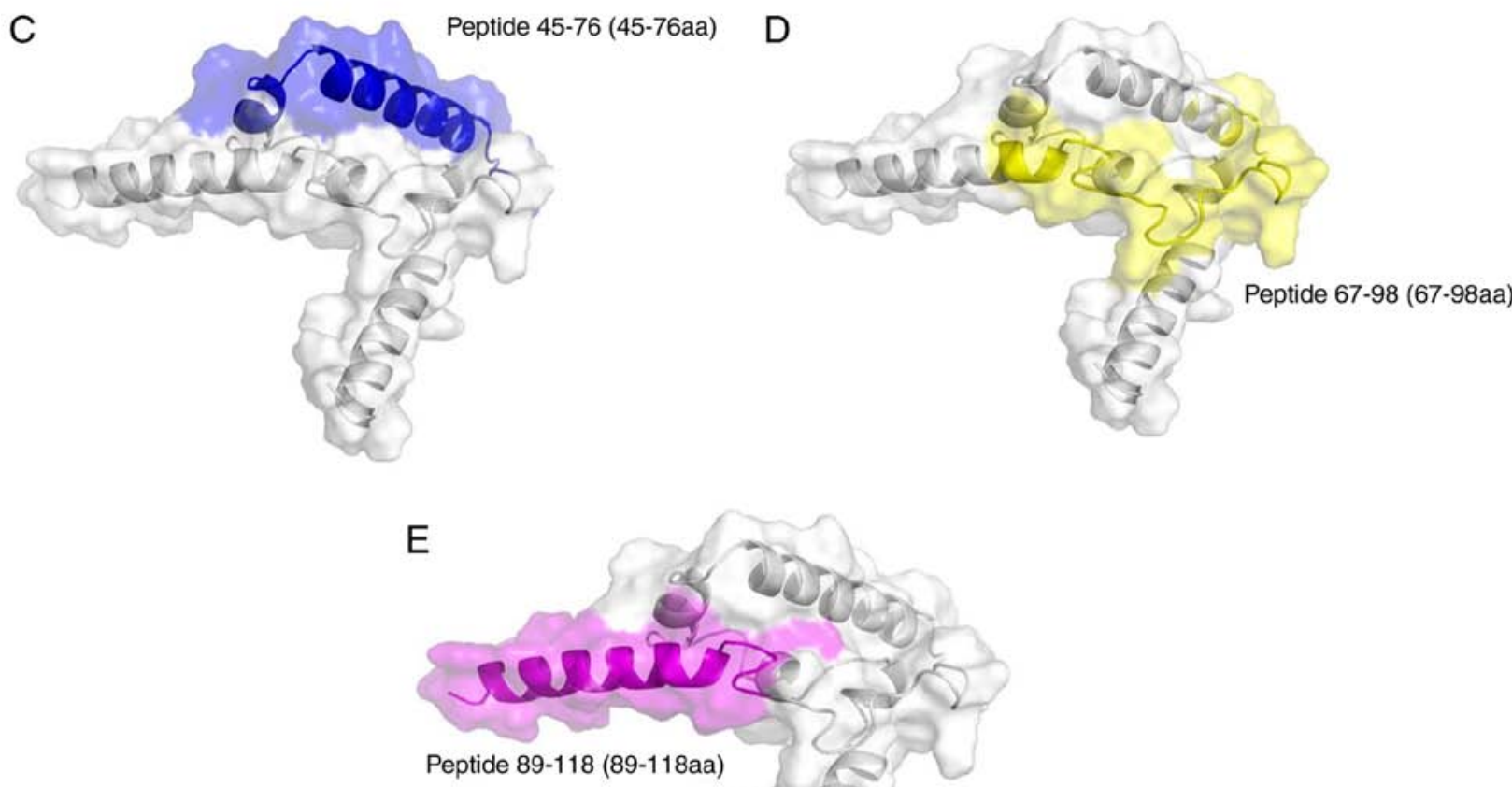

Peptide 89-118 (89-118aa)

Figure 5. Models of synthetic peptide regions in Der f 24. Five synthetic peptide domains are color-coded in the Der f 24 model as follows: (A) peptide 1-32 aa in red, the N-terminal region; (B) peptide 23-54 aa in green; (C) peptide 45-76 aa in blue; (D) peptide 67-98 aa in yellow; and (E) peptide 89-118 aa in magenta. Der $\mathrm{24}$, group 24 allergen of Dermatophagoides farina; aa, amino acid.

presented in Fig. 2A. IgE-western blotting was performed on these proteins by probing with sera pooled from HDM-allergic patients. All of the recombinant proteins except for Der $\mathrm{f}$ 24-Hyb2, which lacked the N-terminal 59-residue region of Der f 24, showed positive IgE-binding (Fig. 2B and C).

Identification of the Der $f 24$ B cell epitope in the $N$-terminal 32-residue region. The IgE epitopes of Der $\mathrm{f} 24$ were further investigated using overlapping synthetic polypeptides coupled with BSA at the N- or C-terminus (Fig. 3A; Table II). IgE-ELISA results revealed that the 1-32 aa peptide group had a stronger binding to HDM allergic-serum IgE compared with that for the other four groups (Fig. 3B). Likewise, IgE-dot blotting showed that the BSA-1-32 aa group had a robust positive reaction to pooled sera from 10 allergic individuals, while the other groups did not have a strong positive reaction (Fig. 3C and D). The rDer f 24 protein was subjected to SDS-PAGE and transferred to PVDF membrane for competitive IgE western blotting. With the addition of increasing concentrations of the BSA-1-32aa conjugate, the capacity of rDer f 24 binding to IgE from HDM allergic sera became weaker (Fig. 3E). These results suggested that the B cell epitope of Der f 24 may be located mainly in the $\mathrm{N}$-terminal 32-residue region.

Peptide 1-32 a domain of Der $f 24$ produces high antibody titers in sensitized mice. To further explore B cell epitope immunogenicity, the titers of IgGs or IgEs targeting the BSA-1-32 aa conjugate were detected mice sensitized with OVA- or OVA-1-32aa conjugate, as OVA induces a 
Table III. BepiPred Linear Epitope Prediction scores of each residue in the $\mathrm{N}$-terminus peptide of $\operatorname{Der} \mathrm{f} 24$ (residues 1-32).

\begin{tabular}{|c|c|c|}
\hline Position & Amino acid & Score \\
\hline 1 & M & -1.518 \\
\hline 2 & $\mathrm{~V}$ & -1.238 \\
\hline 3 & $\mathrm{H}$ & -0.99 \\
\hline 4 & $\mathrm{~L}$ & -1.215 \\
\hline 5 & $\mathrm{~T}$ & -1.172 \\
\hline 6 & K & -1.325 \\
\hline 7 & $\mathrm{~T}$ & -1.327 \\
\hline 8 & $\mathrm{~L}$ & -1.042 \\
\hline 9 & $\mathrm{R}$ & -0.495 \\
\hline 10 & $\mathrm{~F}$ & -0.401 \\
\hline 11 & I & -0.221 \\
\hline 12 & $\mathrm{~N}$ & -0.383 \\
\hline 13 & $\mathrm{~N}$ & -0.063 \\
\hline 14 & $\mathrm{P}$ & 0.007 \\
\hline 15 & G & 0.076 \\
\hline 16 & $\mathrm{~F}$ & 0.234 \\
\hline 17 & $\mathrm{R}$ & -0.095 \\
\hline 18 & $\mathrm{~K}$ & -0.281 \\
\hline 19 & $\mathrm{~F}$ & -0.628 \\
\hline 20 & Y & -0.694 \\
\hline 21 & $\mathrm{Y}$ & -0.489 \\
\hline 22 & G & -0.488 \\
\hline 23 & $\mathrm{~L}$ & -0.308 \\
\hline 24 & Q & 0.104 \\
\hline 25 & G & 0.131 \\
\hline 26 & $\mathrm{Y}$ & 0.347 \\
\hline 27 & $\mathrm{~N}$ & 0.024 \\
\hline 28 & $\mathrm{~K}$ & 0.129 \\
\hline 29 & $\mathrm{Y}$ & -0.004 \\
\hline 30 & G & -0.131 \\
\hline 31 & $\mathrm{~L}$ & -0.169 \\
\hline 32 & Y & -0.487 \\
\hline
\end{tabular}

Der f 24, group 24 allergen of Dermatophagoides farina.

Th2 response in vivo. At 3 weeks post-sensitization, the OVA-1-32 aa group produced high specific $\operatorname{IgG}$ and $\operatorname{IgE}$ antibody titers against the BSA-1-32 aa (Fig. 4A and B), confirming a role for the N-terminal 1-32 region of Der f 24 in IgE-binding activity. Importantly, serum from mice immunized with OVA-1-32aa, but not OVA-injected mice, promoted significant release of $\beta$-hexosaminidase from the mast cell line, RBL-2H3 with the addition of BSA-1-32 aa conjugate (Fig. 4C). Additionally, a competitive IgE-ELISA showed that pooled serum samples of the OVA-1-32 aa-sensitized mice reduced the ability of $\operatorname{IgE}$ in serum pooled from $10 \mathrm{HDM}$ allergic patients to bind to the BSA-1-32 aa antigen (Fig. 4D). These data indicated that the 1-32 aa domain of Der $\mathrm{f} 24$ contains the immunodominant IgE epitopes of Der $f 24$.
Structural location analysis of IgE epitope of Der $f 24$. Superimposing the 3D structural model of Der f 24 on a bovine QCR7 template revealed sequence identity between the template and Der f 24 of $46.60 \%$. Ramachandran plots and RMSD assessment showed that none of the residues were present in the disallowed region, 88 residues were present in the most favored regions, and the overall G-factor of the Der $\mathrm{f}$ 24 structure model was -0.01 (Fig. S1). In addition, structural analysis of homology modelling using the SWISS-MODEL system showed that the 3D structure of Der f 24 was highly similar to that of the QCR7 template (Fig. S2). The RMSD assessment indicated that there were 118 aligned residues in the superimposition (RMSD value, 0.207). These results indicate that the backbone dihedral angles, phi and psi, of the 3D model were within the acceptable range.

Homology modeling in the SWISS-MODEL software revealed that the secondary structure of Der f 24 consisted of three $\alpha$-helices and a free loop (Fig. 5). The structure was positioned on the outside of whole folded protein. This external location may facilitate IgE-binding. According to the results of IEDB prediction, lysine (Lys) 28 and tyrosine (Tyr) 29 residues were located on the $\alpha$-helices and were important for antigen-binding (Table III; Fig. S3). As the peptide 1-32 aa region (red domain in Fig. 5) of Der f 24 was located in a protruding region of the structure, the $\mathrm{N}$-terminal 32-residue region may be more easily accessible for binding by specific IgEs.

\section{Discussion}

In the present study, the immunodominant IgE epitope of Der f 24 was identified using IgE-binding activity assays with Der f-human UQCRB hybrid proteins. The N-terminal region of Der $\mathrm{f} 24$ displayed strong IgE-binding activity and induced high titers of specific IgGs and IgEs in sensitized mice. Additionally, the N-terminal region of Der f 24 induced IgE production in vivo. Homology modeling revealed the structural orientation of this B cell epitope of Der $\mathrm{f} 24$. The identification of the immunodominant IgE epitope of Der $\mathrm{f} 24$ has important implications for HDM allergy diagnosis and treatment.

As allergen-specific IgE antibodies play a crucial role in original immune responses, IgE epitopes of allergens have been investigated (32). The B cell epitope of the allergen can be defined in a linear or conformational form $(33,34)$. Although an allergen may have multiple types of epitopes, IgE-binding of an allergen is highly dependent on its immunodominant epitope (35). For example, the Der p 5 allergen has the linear sequence 90-DRLMQRKDLDIFEQYNLEM-108 and consists conformationally of a bundle of three anti-parallel $\alpha$-helices (36). Although the Group 11 allergenic Blomia tropicalis paramyosin protein contains multiple IgE- and IgG-immunoreactive regions scattered throughout the molecule, its dominant IgE- and IgG-binding regions have been mapped to the aa positions 336-557 and 698-875, respectively (37). Based on the present results, it is reasonable to conclude that Der f 24 also has multiple IgE-binding epitopes, of which the immunodominant IgE epitope would be important in HDM allergy development, and therefore was investigated in this study.

The ubiquitous presence of UQCRB homologs across diverse species, including HDMs and humans, suggest a highly 
conserved biological functionality (38). Der $\mathrm{f} 24$ was found to be highly homologous to Der $\mathrm{p} 24$, but to share a relatively low sequence homology with human UQCRB, which was expected based on the selective allergenicity of the HDM UQCRB homologs. Conversely, human and mouse UQCRB were found to be highly homologous. Among the hybrid proteins tested for IgE-binding in the current study, only the Der f 24-Hyb2 protein that lacked the N-terminal 59-residue region of Der f 24 was not immunoreactive. All of the other synthetic hybrid protein variants were immunoreactive, suggesting strongly that the B cell epitope of Der f 24 is located, at least mostly, in the N-terminal 59-residue region, where a $37.29 \%$ sequence homology (22 out of 59 aa) was found between Der $\mathrm{f}$ and human UQCRB. This sequence divergence is likely to underlie, at least to some extent, the IgE-binding activity of Der $\mathrm{f} 24$. In the present study, activity assays using the rDer f 24 protein as a UQCRB-like protein were hard to perform due to quality control of the recombinant protein. The rDer $f$ 24 protein required a positive rate of $\sim 50 \%$ using 10 individual HDM allergic serum samples for IgE-ELISA according to our previous study (9).

The identification of IgE immunodominant epitopes of HDM allergens can elucidate the mechanisms of allergen-induced sensitization and provide useful information for the prevention and treatment of allergic diseases. A study by Greene and Thomas (39) found five Der p 1 linear IgE epitopes (aa 15-33, 60-80, 81-94, 101-111 and 155-187). Furthermore, a study by Van't Hof et al (40) identified an immunodominant IgE epitope in Der $\mathrm{p} 2$ that was located near the $\mathrm{N}$ terminus (aa 65-78), similar to the IgE epitope in Der $\mathrm{f} 24$ identified in the present study. Previously, peptide-displaying phage technology and artificial synthesized peptide scanning technology have been used to identify immunodominant epitopes $(37,40)$. Additionally, our previous study showed that the immunodominant IgE epitopes of Der p 24 located in the N-terminal 32-residue region triggered IgE production in vivo (25). In the present study, artificial hybrid proteins and synthesized polypeptides were used to identify the N-terminal region of Der $\mathrm{f}$ 24 as a major mediator of the IgE-binding activity of Der $f 24$.

OVA can be used as a carrier protein, like BSA, to enhance immune response activation. Unlike BSA, OVA generates a strong Th2 response in vivo (41). The $\mathrm{N}$-terminal 1-32 aa region of the Der $\mathrm{f} 24-\mathrm{OVA}$ conjugates produced specific IgE responses in vivo in the current study. Notably, structural modeling showed that the N-terminal 32 residues of Der $\mathrm{f} 24$ form a structural protrusion that may be conducive to specific IgE-binding. Mutation experiments are required to investigate the importance of the Lys 28 and Tyr 29 residues of Der $\mathrm{f} 24$ for IgE-binding. The results of the present study were similar to that of homolog Der p 24, indicating that the major allergen Group 24 in HDM may share a similar dominant IgE epitope and produce similar clinical significance (25).

At present, allergen immunotherapy is an effective way of treating IgE-mediated allergies (42). As a single epitope often may not perform well for allergic diagnosis or therapy, HDM whole protein extract is the preferred choice during clinical application (7). Identification of more immunodominant IgE epitopes of HDM allergens is required for further refinement. Hence, elucidation of the IgE epitopes of allergens can serve to facilitate the rational design of hypoallergenic candidate vaccine immunotherapies for allergies (43). Natural HDM allergen extracts and vaccines based on these complex molecular extracts are known to produce adverse secondary effects $(44,45)$. Genetic recombinant hybrid proteins have emerged as another approach to immunotherapy development (46). Good outcomes of such immunotherapy approaches are dependent upon clarification of immunodominant epitopes of allergens (45). Carrier-bound HDM Group 1 allergen-derived peptides have been produced for HDM allergen immunotherapy development (47). A previous study demonstrated that recombinant hybrid proteins consisting of reassembled Der $\mathrm{p} 1$ and Der $\mathrm{p} 2$ fragments can be used as a safe hypoallergenic molecule for achieving HDM allergen tolerance and vaccination (2). Thus, the identification of the immunodominant epitope of Der f 24 contributes to the theoretical foundation for developing a hypoallergenic vaccine for HDM allergies.

In conclusion, the results of the present study suggest that the immunodominant IgE epitope of Der $\mathrm{f} 24$ is located mainly in the $\mathrm{N}$-terminal 32 aa region. These findings may help to elucidate the mechanisms of HDM allergy sensitization. Based on the finding of the current study and previously published studies, HDM allergens have the potential to induce allergic reactions by way of protein functions or by interaction with IgE B cell epitopes.

\section{Acknowledgements}

We thank the other members of Kunmei Ji's lab for their critical comments and, we thank the Department of Allergy and Clinical Immunology of the First Affiliated Hospital of Guangzhou Medical College (Guangdong, China) for supporting serum sample collection.

\section{Funding}

The present study was supported in part by research funding from the National Natural Science Foundation of China (grant. no. 81571570), Guangdong Province (grants. no. 2016A020215176, 2016A030313039, 2017A010105014 and 2018A050506083), and Shenzhen City 2016 Biochemistry Discipline Construction.

\section{Availability of data and materials}

The datasets used and/or analyzed during the current study are available from the corresponding author on reasonable request.

\section{Authors' contributions}

CJJ and JKM conceived and designed the study. ZLC and JJC analyzed the data. WLL, ZLC, ZZ, YBH and YSH performed the studies. JJC and $\mathrm{KJ}$ contributed reagents materials and analysis tools. JJC, ZZ and $\mathrm{KJ}$ wrote the manuscript.

\section{Ethics approval and consent to participate}

Permission to conduct this study was obtained from the Ethics Committee of the First Affiliated Hospital of Guangzhou Medical College (no. 2012-51). Informed consent was obtained 
from all individual participants included in the study. All procedures involving human participants were in accordance with the ethical standards of the committee.

\section{Patient consent for publication}

Not applicable.

\section{Competing interests}

The authors declare that they have no competing interests.

\section{References}

1. D'Amato G,LiccardiG,D'Amato M and Holgate S: Environmental risk factors and allergic bronchial asthma. Clin Exp Allergy 35: 1113-1124, 2005

2. Ciprandi G, Puccinelli P, Incorvaia C and Passalacqua G; Italian Cometa Study Group: The relevance of house dust mites allergy in clinical practice: The epidemiological impact on allergen immunotherapy. Immunotherapy 9: 1219-1224, 2017.

3. Bousquet PJ, Chinn S, Janson C, Kogevinas M, Burney P and Jarvis D; European Community Respiratory Health Survey I: Geographical variation in the prevalence of positive skin tests to environmental aeroallergens in the European community respiratory health survey I. Allergy 62: 301-309, 2007.

4. Chew GL, Reardon AM, Correa JC, Young M, Acosta L, Mellins R, Chew FT and Perzanowski MS: Mite sensitization among Latina women in New York, where dust-mite allergen levels are typically low. Indoor Air 19: 193-197, 2009.

5. Yang L and Zhu R: Immunotherapy of house dust mite allergy. Hum Vaccin Immunother 13: 2390-2396, 2017.

6. Pomés A, Davies JM, Gadermaier G, Hilger C, Holzhaouser T, Lidholm J, Lopata AL, Mueller GA, Nandy A, Radauer C, et al: WHO/IUIS allergen nomenclature: Providing a common language. Mol Immunol 100: 3-13, 2018.

7. Thomas WR, Smith WA, Hales BJ, Mills KL and O'Brien RM: Characterization and immunobiology of house dust mite allergens. Int Arch Allergy Immunol 129: 1-18, 2002.

8. Weghofer M, Grote M, Resch Y, Casset A, Kneidinger M, Kopec J, Thomas WR, Fernández-Caldas E, Kabesch M, Ferrara R, et al: Identification of Der $\mathrm{p} 23$, a peritrophin-like protein, as a new major Dermatophagoides pteronyssinus allergen associated with the peritrophic matrix of mite fecal pellets. J Immunol 190: 3059-3067, 2013

9. Chan TF, Ji KM, Yim AK, Liu XY, Zhou JW, Li RQ, Yang KY, Li J, Li M, Law PT, et al: The draft genome, transcriptome, and microbiome of Dermatophagoides farinae reveal a broad spectrum of dust mite allergens. J Allergy Clin Immunol 135: 539-548, 2015.

10. Gough L, Schulz O, Sewell HF and Shakib F: The cysteine protease activity of the major dust mite allergen Der $\mathrm{p} 1$ selectively enhances the immunoglobulin E antibody response. J Exp Med 190: 1897-902, 1999.

11. Gough L, Sewell HF and Shakib F: The proteolytic activity of the major dust mite allergen Der 1 enhances the IgE antibody response to a bystander antigen. Clin Exp Allergy 31: 1594-1598, 2001.

12. Trompette A, Divanovic S, Visintin A, Blanchard C, Hegde RS, Madan R, Thorne PS, Wills-Karp M, Gioannini TL, Weiss JP and Karp CL: Allergenicity resulting from functional mimicry of a Toll-like receptor complex protein. Nature 457: 585-588, 2009.

13. Park BS, Lee NR, Kim MJ, Kim SY and Kim IS: Interaction of Der p 2 with Toll-like receptor 4 and its effect on cytokine secretion. Biomed Sci Letters 21: 152-159, 2015.

14. Hales BJ, Martin AC, Pearce LJ, Laing IA, Hayden CM, Goldblatt J, Le Souef PN and Thomas WR: IgE and IgG anti-house dust mite specificities in allergic disease. J Allergy Clin Immunol 118: 361-367, 2006.

15. Jacquet A: Innate immune responses in house dust mite allergy. ISRN Allergy 2013: 735031, 2013.

16. Amin K: The role of mast cells in allergic inflammation. Respir Med 106: 9-14, 2012.

17. Pomes A: Relevant B cell epitopes in allergic disease. Int Arch Allergy Immunol 152: 1-11, 2010.

18. Matsuo H, Yokooji $\mathrm{T}$ and Taogoshi T: Common food allergens and their IgE-binding epitopes. Allergol Int 64: 332-343, 2015.
19. Bronnert M, Mancini J, Birnbaum J, Agabriel C, Liabeuf V, Porri F, Cleach I, Fabre A, Deneux I, Grandné V, et al: Component-resolved diagnosis with commercially available D. pteronyssinus Der p 1, Der p 2 and Der p 10: Relevant markers for house dust mite allergy. Clin Exp Allergy 42: 1406-1415, 2012.

20. Chou H, Tam MF, Lee SS, Tang RB, Lin TH, Tai HY, Chen YS and Shen HD: Asp159 is a critical core amino acid of an IgE-binding and cross-reactive epitope of a dust mite allergen Der f 7. Mol Immunol 48: 2130-2134, 2011

21. Chruszcz M, Chapman MD, Vailes LD, Stura EA, Saint-Remy JM, Minor W and Pomes A: Crystal structures of mite allergens Der $f 1$ and Der p 1 reveal differences in surface-exposed residues that may influence antibody binding. J Mol Biol 386: 520-530, 2009.

22. de Halleux S, Stura E, VanderElst L, Carlier V, Jacquemin M and Saint-Remy JM: Three-dimensional structure and IgE-binding properties of mature fully active Der $\mathrm{p} 1$, a clinically relevant major allergen. J Allergy Clin Immunol 117: 571-576, 2006.

23. Takai T, Kato T, Yasueda H, Okumura K and Ogawa H: Analysis of the structure and allergenicity of recombinant pro- and mature Der $\mathrm{p} 1$ and Der f 1: Major conformational IgE epitopes blocked by prodomains. J Allergy Clin Immunol 115: 555-563, 2005.

24. Cho YS, Jung HJ, Seok SH, Payumo AY, Chen JK and Kwon HJ: Functional inhibition of UQCRB suppresses angiogenesis in zebrafish. Biochem Biophys Res Commun 433: 396-400, 2013.

25. Cai ZL, Chen JJ, Zhang Z, Hou YB, He YS, Sun JL and Ji K: Identification of immunodominant IgE binding epitopes of Der p 24, a major allergen of Dermatophagoides pteronyssinus. Clin Transl Allergy 9: 28, 2019.

26. Chen ZG, Li YT, Wang WH, Tan KS, Zheng R, Yang LF, Guan WJ, Hong HY and Yang QT: Distribution and determinants of dermatophagoides mites sensitization of allergic rhinitis and allergic asthma in China. Int Arch Allergy Immunol 180: 17-27, 2019.

27. Thomas WR: Geography of house dust mite allergens. Asian Pac J Allergy Immunol 28: 211-224, 2010.

28. Liu XY, Ji KM, Gao B and Liu ZG: Expression, purification and identification of the recombinant allergen Der p2 from Dermatophagoides pteronyssinus and investigation on its immunological activities. Chin J Zoonoses 009, 929-935, 2009.

29. Lauber B, Molitor V, Meury S, Doherr MG, Favrot C, Tengvall K, Bergvall K, Leeb T, Roosje P and Marti E: Total IgE and allergen-specific IgE and IgG antibody levels in sera of atopic dermatitis affected and non-affected Labrador- and Golden retrievers. Vet Immunol Immunopathol 149: 112-118, 2012.

30. Taylor JA, Karas JL, Ram MK, Green OM and Seidel-Dugan C: Activation of the high-affinity immunoglobulin $\mathrm{E}$ receptor $\mathrm{Fc}$ epsilon RI in RBL-2H3 cells is inhibited by Syk SH2 domains. Mol Cell Biol 15: 4149-4157, 1995.

31. Zhang Q, Wang P, Kim Y, Haste-Andersen P, Beaver J, Bourne PE, Bui HH, Buus S, Frankild S, Greenbaum J, et al: Immune epitope database analysis resource (IEDB-AR). Nucleic Acids Res 36 (Web Server Issue): W513-W518, 2008.

32. Costa MA, Duro G, Izzo V, Colombo P, Mirisola MG, Locorotondo G, Cocchiara R and Geraci D: The IgE-binding epitopes of $\mathrm{rPar} \mathrm{j} 2$, a major allergen of Parietaria judaica pollen, are heterogeneously recognized among allergic subjects. Allergy 55: 246-250, 2000

33. Banerjee B, Greenberger PA, Fink JN and Kurup VP: Conformational and linear B-cell epitopes of Asp $\mathrm{f} 2$, a major allergen of Aspergillus fumigatus, bind differently to immunoglobulin $\mathrm{E}$ antibody in the sera of allergic bronchopulmonary aspergillosis patients. Infect Immun 67: 2284-2291, 1999.

34. Szalai K, Fuhrmann J, Pavkov T, Scheidl M, Wallmann J, Brämswig KH, Vrtala S, Scheiner O, Keller W, Saint-Remy JM et al: Mimotopes identify conformational B-cell epitopes on the two major house dust mite allergens Der p 1 and Der p 2. Mol Immunol 45: 1308-1317, 2008.

35. Stanley JS, King N, Burks AW, Huang SK, Sampson H, Cockrell G, Helm RM, West CM and Bannon GA: Identification and mutational analysis of the immunodominant IgE binding epitopes of the major peanut allergen Ara h 2. Arch Biochem Biophys 34: 244-253, 1997.

36. Laskowski RA, MacArthur MW, Moss DS and Thornton JM: PROCHECK-a program to check the stereochemical quality of protein structures. J App Cryst 26: 283-291, 1993.

37. Ramos JD, Cheong N, Lee BW and Chua KY: Peptide mapping of immunoglobulin $\mathrm{E}$ and immunoglobulin $\mathrm{G}$ immunodominant epitopes of an allergenic Blomia tropicalis paramyosin, Blo $\mathrm{t} 11$. Clin Exp Allergy 33: 511-517, 2003. 
38. Jung HJ, Kim KH, Kim ND, Han G and Kwon HJ: Identification of a novel small molecule targeting UQCRB of mitochondrial complex III and its anti-angiogenic activity. Bioorg Med Chem Lett 21: 1052-1056, 2011.

39. Greene WK and Thomas WR: IgE binding structures of the major house dust mite allergen Der p I. Mol Immunol 29: 257-262, 1992

40. Van't Hof W, Driedijk PC, van den Berg M, Beck-Sickinger AG, Jung Gand Aalberse RC: Epitope mapping of the Dermatophagoides pteronyssinus house dust mite major allergen Der $\mathrm{p}$ II using overlapping synthetic peptides. Mol Immunol 28: 1225-1232, 1991.

41. Herrick CA, MacLeod H, Glusac E, Tigelaar RE and Bottomly K: $\mathrm{Th} 2$ responses induced by epicutaneous or inhalational protein exposure are differentially dependent on IL-4. J Clin Invest 105: $765-775,2000$.

42. Moingeon P and Mascarell L: Novel routes for allergen immunotherapy: Safety, efficacy and mode of action. Immunotherapy 4: 201-212, 2012.

43. Chen KW, Blatt K, Thomas WR, Swoboda I, Valent P, Valenta R and Vrtala S: Hypoallergenic Der p 1/Der p 2 combination vaccines for immunotherapy of house dust mite allergy. J Allergy Clin Immunol 130: 435-443, 2012.
44. Winther L, Arnved J, Malling HJ, Nolte $\mathrm{H}$ and Mosbech $\mathrm{H}$ : Side-effects of allergen-specific immunotherapy: A prospective multi-centre study. Clin Exp Allergy 36: 254-260, 2006.

45. Nishiyama C, Fukada M, Usui Y, Iwamoto N, Yuuki T, Okumura Y and Okudaira H: Analysis of the IgE-epitope of Der $\mathrm{f} 2$, a major mite allergen, by in vitro mutagenesis. Mol Immunol 32: 1021-1029, 1995.

46. Ferreira F, Wallner M, Breiteneder H, Hartl A, Thalhamer J and Ebner C: Genetic engineering of allergens: Future therapeutic products. Int Arch Allergy Immunol 128: 171-178, 2002.

47. Fanuel S, Tabesh S, Mokhtarian K, Saroddiny E, Fazlollahi MR, Pourpak Z, Falak R and Kardar GA: Construction of a recombinant B-cell epitope vaccine based on a Der p1-derived hypoallergen: A bioinformatics approach. Immunotherapy 10: 537-553, 2018 .

(i) $(\Theta$ This work is licensed under a Creative Commons Attribution-NonCommercial-NoDerivatives 4.0 International (CC BY-NC-ND 4.0) License. 